\title{
INFLUÊNCIA DA ÊPOCA DE SELEÇÃO DO REBENTO SOBRE O DESENVOLVIMENTO E PRODUÇÃO DA PLANTA MATRIZ DE BANANEIRA (MUSA CAVENDISHII) CULTIVAR NANICÃO *
}

\author{
Ivo Manica \\ Freddy P. Zambrano Peres \\ Salim Simão
}

\section{RESUMO}

\begin{abstract}
No presente trabalho foram feitos os tratamentos: planta matriz sem rebentos; planta matriz com rebentos de janeiro, marco e maio e testemunha com todos os rebentos, sendo estudada sua influência no desenvolvimento e produção da planta matriz de bananeira.

Os resultados mostraram diferenca significativa entre os tratamentos, com relação ao número de dias do plantio ao florescimento, ciclo de produção, número de folhas na colheita e peso do cacho. Não houve diferença significativa em relação ao diâmetro do pseudocaule no florescimento e colheita, número de pencas e frutos.
\end{abstract}

\section{INTRODUÇÃO}

O Brasil é o primeiro produtor mundial de bananas, com área cultivada de 273.000 hectares e produção de 6.023 .000 ton. métricas de frutos em 1968, conforme a F. A. O. (1970).

A bananeira ocupava também, em 1969, o $1 .^{\circ}$ lugar entre as fruteiras cultivadas em nosso país, com 273.113 hectares de área cultivada e valor da produção calculado em Cr\$565.245.000,00, segundo o I. B. G. E. (1972).

$\mathrm{O}$ ciclo completo da bananeira, do plantio à colheita do cacho, varia com o clima da região, solo e tratos culturais, sendo aproximadamente de 12 a 18 meses.

Atualmente, em explorações bem orientadas, a tendência é conduzir o bananal com uma planta matriz e apenas um ou dois rebentos que saem em

\footnotetext{
* Entregue para publicação em 21/12/1973.

** Departamento de Fitotecnia - Universidade de Viçosa, MG.

*** Universidade Central Universal Ocidental - Barquisimetro - Venezuela.

**** Departamento de Agricultura e Horticultura - ESALQ - Piracicaba - S. P.
} 
épocas diferentes, com a finalidade de manter na mesma cova 2 a 3 plantas em diferentes estádios de desenvolvimento.

Como a seleção de rebentos depende da época de plantio, tipo de muda, clima, solo e tratos culturais, uma planta pode produzir frutos praticamente durante todo o ano, existindo porém, em São Paulo, tendência a maior produção de cachos de janeiro a julho e menor de agosto a dezembro.

A maior ou menor oferta de bananas reflete no preço do produto no mercado, sendo que uma época de menor produção corresponde a um maior preço e uma época de maior produção a um menor preço.

O presente trabalho estuda a influência da época de seleção do rebento sobre o desenvolvimento e produção da planta matriz da bananeira nanicão.

\section{REVISÃO DE LITERATURA}

A grande aplicação do desbaste, segundo KERVEGANT (1935) é regular a época da colheita do cacho, porém, é necessário bastante conhecimento para selecionar rebentos que frutifiquem em períodos favoráveis, pois os mesmos são muito influenciados pelo clima, solo e exposição. Para AUBERT (1971), a produção de bananas pode ser ajustada em função das condições climáticas e econômicas, através da seleção do material de plantio, desbaste dos rebentos e época de plantio.

RODRIGUES E SOUZA (1947), trabalhando com o cultivar Nanica, verificaram que dois fatores intervém na distribuição da colheita durante os meses do ano: seleção dos rebentos e duração do período de atividade vegetativa.

CHAMPION (1963), com relação a clima, considera a atividade vegetativa da bananeira muito fraca com temperaturas inferiores a $16^{\circ} \mathrm{C}$. Temperaturas em torno de $25^{\circ} \mathrm{C}$ e chuvas de 100 a $120 \mathrm{~mm}$. mensais, são as que podem proporcionar um excelente desenvolvimento à bananeira.

WARDLAW (1961), observou que com a ocorrência de temperaturas de $25,5^{\circ} \mathrm{C}$ dois meses antes da colheita, aumentava muito o peso do cacho. Temperaturas mais altas aceleravam a maturação e temperaturas mais baixas prejudicavam o desenvolvimento do fruto.

CORTEZ (1961), em ensaio realizado no Litoral de São Paulo, verificou, que a qualidade do fruto (número de pencas, frutos e peso) dependia do período em que se dava a diferenciação floral e florescimento.

SAMPAIO (1967), utilizando o cultivar Nanicão, obteve uma média de 12,1 folhas por planta; 7,3 pencas por cacho, e ciclo de 289,1 dias do plantio ao florescimento; BORGES (1971), na Venezuela, obteve plantas que alcançaram uma altura média de $216 \mathrm{~cm}$, tiveram ciclo de 215 dias do plantio ao florescimento e produziram uma média de 7 pencas por cacho.

MATTOS(1969), em plantio com espaçamento de 2,0 x 2,5 m obteve um diâmetro de pseudo-caule de $18,2 \mathrm{~cm}$, altura da planta de $208,1 \mathrm{~cm} ; 12,2$ folhas por planta, 7,5 pencas por cacho, peso do cacho de $16,5 \mathrm{~kg}$ e ciclo de produção de 488 dias. 
AHMED e MANNAN (1971) empregaram diferentes espaçamentos de plantio, em covas de 30,45 e $60 \mathrm{~cm}$ cúbicos. O pseudo-caule alcançou menor diâmetro e uma produção de frutos de 24,6 e $104,2 \%$ a mais por unidade de área no espaçamento de $1,2 \times 1,2 \mathrm{~m}$ do que nos espaçamentos de $1,8 \times 1,8 \mathrm{~m}$ e $2,4 \times 2,4 \mathrm{~m}$. As plantas com espaçamento de $1,2 \times 1,2 \mathrm{~m}$ produziram $63,8 \%$ a mais de frutos do que as espaçadas de $2,4 \times 2,4 \mathrm{~m}$. O maior espaçamento apresentou cachos, mãos e dedos mais pesados. 0 florescimento e a maturação dos frutos foi retardada no espaçamento menor.

O espaçamento de $1,8 \times 1,8 \mathrm{~m}$, com covas no tamanho de $60 \mathrm{~cm}$ cúbicos constituiu a combinação mais econômica.

MATTOS (1969) utilizou espaçamentos de $2 ; 0 \times 1,5 \mathrm{~m} ; 2,0 \times 2,0 ; 2,0 \times 2,5$; $2,0 \times 3,0 ; 3,0 \times 2,5 ; 3,0 \times 3,0$. O primeiro ciclo de produção não apresentou diferença significativa entre os plantios em relação ao diâmetro do pseudo-caule, número de folhas ativas e pencas; porém em relação ao peso do cacho e ciclo de produção, os espaçamentos de $3,0 \times 2,0$ e $3,0 \times 3,0$ m. mostraram diferença significativa quando comparados com 0 espaçamento de $2,0 \times$ $1,5 \cdot \mathrm{m}$.

MISSINGHAN (1963) em plantios com espaçamentos de $4 \mathrm{~m}$.entre filas e $0,9 \mathrm{~m} ; 1,2 \mathrm{~m} ; 1,5 ; 1,8 \mathrm{~m}$; e $2,1 \mathrm{~m}$. dentro da mesma fila verificou que o espaçamento teve pouco efeito no tamanho do cacho e no tempo decorrido do plantio à colheita da planta matriz. Os frutos de todos os tratamentos apresentaram boa qualidade, porém na produção do primeiro rebento, os espaçamentos menores produziram cachos menores e com péssimo enchimento de frutos.

Espaçamentos de $1,8 \times 1,2 \mathrm{~m} ; 1,8 \times 1,8 \mathrm{~m}$ e $1,8 \times 2,4 \mathrm{~m}$., estudados por JAGIRDAR et alii (1964), não mostraram influência no rendimento, mas os espaçamentos maiores produziram maior número de rebentos.

BERRIL (1963) efetuou plantios em linhas distanciadas de 2,7 m. 1,2 a $3,0 \mathrm{~m}$. dentro da mesma linha. Obteve na primeira colheita maior taxa de crescimento, maior número de rebentos e florescimento mais precoce com o maior espaçamento; porém o rendimento de frutos por hectare foi maior utilizando o espaçamento $1,2 \times 2,7 \mathrm{~m}$.. Não foi possível controlar os rebentos da segunda colheita, no espaçamento de $1,2 \times 2,7 \mathrm{~m}$.. O máximo rendimento, para a primeira e segunda colheitas, foi obtido com espaçamentos de $1,5 \mathbf{x}$ 2,7 m., correspondendo a uma densidade de 2.445 plantas por hectare.

Para as variedades Mantmann e Champa, com plantio feito no espaçamento de 2,7 x 2,7 m., BHAN e MAZUNDER (1961) obtiveram um rendimento de 77 e $66,4 \%$ a mais por unidade de área do que em plantios feitos no espaçamento de $3,6 \times 3,6 \mathrm{~m}$. A Nanica produziu $78,6 \%$ mais frutos com 1,8 $x 1,8 \mathrm{~m}$. do que com espaçamento de $2,4 \times 2,4 \mathrm{~m}$. Espaçamentos menores não diminuiram o peso médio dos cachos nas variedades Mantaman e Nanica, porém a Champa mostrou redução significativa no $3 .^{\circ}$ rebento. $O$ número de pencas e frutos não foi influenciado pelos espaçamentos em nenhuma variedade. 


\section{MATERIAL E MÉTODOS}

O presente ensaio foi instalado em Área do Departamento de Agricultura e Horticultura da Escola Superior de Agricultura «Luiz de Queiroz», em Piracicaba, em solo pertencente, segundo RANZANI ef alii (1969), à série «Luiz de Queiroz». O clima da região é mesotérmico, úmido, subtropical, com inverno seco, designado Cwa, segundo o sistema de classificação de Koopen.

A temperatura média do mês mais quente durante o período do ensaio foi de $24,3^{\circ} \mathrm{C}$ e a do mês mais frio de $16,9^{\circ} \mathrm{C}$; a temperatura máxima foi de $30,4^{\circ} \mathrm{C}$ e a mínima de $9,0^{\circ} \mathrm{C}$. A minima absoluta em junho de 1972 , foi de $3,0^{\circ} \mathrm{C}$ e no mês de julho de $0,2^{\circ} \mathrm{C}$.

Foi utilizado no plantio o cultivar Nanicão (Musa cavendishii Lambert), com mudas do tipo «chifre» e peso médio de $2,3 \mathrm{~kg}$. As mudas foram retiradas do bananal e mergulhadas numa solução contendo $\mathrm{BHC}$ a $5 \%$ pelo tempo de 1 minuto, e posteriormente deixadas secar à sombra durante 6 horas. A área foi arada, gradeada e sulcada de $2,5 \times 2,5 \mathrm{~m}$, com sulcos de $30 \mathrm{~cm}$ de profundidade. Após a sulcagem, foi aplicado no sulco $500 \mathrm{~g}$ de fórmula 10-5-20 de NPK, sendo o adubo misturado com o solo na posicão correspendente a cada muda.

No dia 17 de setembro de 1971 , as mudas foram plantadas na distância de 2 metros dentro do sulco; em 26/01/72 realizou-se uma adubação em cobertura, com $250 \mathrm{~g}$ de sulfato de amônio por planta e em 17/03/1971, com 500 g. da fórmula 10-5-20 NPK; em 17/10/1972 foram aplicados $50 \mathrm{~g}$. de cloreto de potássio, $200 \mathrm{~g}$. de sulfato de amônio e $40 \mathrm{~g}$. de superfosfato triplo por planta, sendo feita a última adubação em 24/01/1973 empregando $100 \mathrm{~g}$. de cloreto de potássio por cova, com aplicação de adubo em coroa ao redor da planta.

No início do experimento, as plantas foram irrigadas em sulcos com intervalos de 7 dias; de maio a agosto as irrigações foram espaçadas de 15 em 15 dias ou feitas 15 dias após as chuvas; a partir de 25/01/72 as irrigações foram feitas sempre que a percentagem de água útil do solo atingia $50 \%$.

Durante o decorrer do experimento, foram efetuados desbastes de rebentos e controle das ervas daninhas.

O delineamento experimental foi feito em blocos inteiramente casualizados, com 5 tratamentos e 5 repetições. Cada parcela era composta de 6 plantas úteis, sendo o total de plantas da mesma parcela em número de 20.

Os tratamentos foram os seguintes:

1. SR - Planta matriz sem rebento;

2. RJ - Planta matriz com seleção do rebento do mês de janeiro;

3. RM - Planta matriz com seleção do rebento do mês de março;

4. RMa - Planta matriz com seleção do rebento do mês de maio;

5. T - Testemunha com todos os rebentos presentes. 
Semanalmente foram feitas anotações do número de folhas, diâmetro do pseudo-caule na época da inflorescência e colheita do cacha; peso, número de pencas e frutos por ocasião da colheita.

\section{RESULTADOS E DISCUSSAOO}

\section{Número de dias do plantio ao florescimento}

A análise de variância para número de dias decorridos do plantio ao florescimento, mostrou diferença significativa entre os tratamentos, ao nível de $5 \%$ de probabilidade.

Comparação das médias dos diversos tratamentos aparece no quadro I.

Quadro 1 - Comparação das médias do número de dias do plantio ao florescimento

Tratamentos

$4 \mathrm{RMa}$ - Planta com rebento de maio

$5 \mathrm{~T}$ - Testemunha com todos os rebentos

2 RJ - Planta com rebento de janeiro

1 SR - Planta sem rebento

3 RM - Planta com rebento de março
Média do número de dias

293,4 a $\left(^{*}\right)$
290,2 b
278,6 b c
273,0 b c
263,8

(*) As médias seguidas por letras diferentes apresentam diferença significativa ao nivel de $5 \%$ de probabilidade, pelo teste de Tukey.

A comparação das médias com relação ao número de dias do plantio ao florescimento, mostrou que os tratamentos $4 \mathrm{RMa}$ e $5 \mathrm{~T}$ foram equivalentes entre si, porém o tratamento $4 \mathrm{RMa}$ diferiu significativamente dos tratamentos $2 \mathrm{RJ}, 1 \mathrm{SR}$ e $3 \mathrm{RM}$; o tratamento $5 \mathrm{~T}$ e $2 \mathrm{RJ}$ e $1 \mathrm{SR}$ foram equivalentes entre si, porém, o tratamento $5 \mathrm{~T}$ apresentou diferença significativa em relação ao tratamento $3 \mathrm{RM}$; os tratamentos $2 \mathrm{RJ}, 1 \mathrm{Sr}$ e $3 \mathrm{RM}$ não se diferenciaram entre si.

0 tratamento com rebento de maio (4 $\mathrm{RMa}$ ) apresentou resultado não esperado. Seu ciclo deveria ser semelhante ao do tratamento sem rebento (1 SR), considerando que seu rebento iniciou o crescimento no mês de maio, época em que grande percentagem das plantas já haviam iniciado o florescimento. $O$ tratamento testemunha com todos os rebentos $(5 \mathrm{~T})$ apresentou o resultado esperado: tendo permanecido com todos os rebentos que nasciam, deve ter ocorrido maior competição entre os rebentos e a planta matriz, e como consequência ocorreu menor desenvolvimento da mesma.

$\mathrm{O}$ número de dias decorridos do plantio ao florescimento, neste ensaio, foi semelhante ao obtido por SAMPAIO (1967), enquanto que BORGES (1971), 
na Venezuela, obteve um ciclo menor. As pesquisas de AHMED e MANNAN (1971) e JAGIRDAR et alii (1964), mostraram um ciclo menor utilizando espaçamentos maiores.

\section{Diâmetro do pseudo-caule na época do aparecimento da inflorescência}

A análise de variância não mostrou diferença significativa entre os diversos tratamentos, com relação ao diâmetro do pseudo-caule na época do aparecimento dà inflorescência; os diâmetros foram 19,2 cm;18,52 cm;18,42 $\mathrm{cm} ; 18,08 \mathrm{~cm}$ e $17,20 \mathrm{~cm}$, para os tratamentos $3 \mathrm{RM}, 4 \mathrm{RMa}, 1 \mathrm{SR}, 5 \mathrm{~T}$ e $2 \mathrm{RJ}$, respectivamente

MATTOS (1969) obteve $18,2 \mathrm{~cm}$, de diâmetro do pseudo-caule, com resultados semelhantes aos obtidos neste trabalho, mas AHMED e MANNAN (1971) obtiveram um menor diâmetro do pseudo-caule utilizando espaçamentos menores.

\section{Número de folhas na época do aparecimento da inflorescência}

A análise de variância para número de folhas na época do aparecimento da inflorescência mostrou diferença significativa entre os tratamentos, ao nível de $5 \%$ de probabilidade.

O quadro 2 apresenta a comparação das médias do número de folhas, na época do aparecimento da inflorescência.

Quadro 2 - Comparação das nédias para número de foilias na infíorescência.

Tratamentos

3 RM

$1 \mathrm{SR}$

4 RMa

$2 \mathrm{RJ}$

$5 \mathrm{~T}$
Média do número de folhas

$$
\begin{aligned}
& 16,94 \text { a }\left(^{*}\right) \\
& 16,90 \text { a } \\
& 16,46 \text { a } \\
& 15,86 \text { b } \\
& 15,32 \text { b }
\end{aligned}
$$

(*) As médias seguidas por letras diferentes apresentam diferença significativa ao nível de $5 \%$ de probabilidade pelo teste de Tukey.

Os tratamentos $3 \mathrm{RM}, 1$ SR e $4 \mathrm{RMa}$ foram equivalentes entre si, porém apresentam diferença significativa em relação aos tratamentos 2 RJ e $5 \mathrm{~T}$ que não se diferenciaram entre si.

A diferença do número de folhas na época do aparecimento da inflorescência, havendo menor número nos tratamentos com rebentos de janeiro (2 RJ) e na testemunha com todos os rebentos $(5 \mathrm{~T})$, pode ser atribuída à 
competição entre a planta matriz e entre os demais rebentos nascidos no tratamento testemunha, e entre a planta matriz e o rebento de janeiro. 0 tratamento sem rebentos (1 SR) ficou durante todo o período de desenvolvimento apenas com a planta matriz, e os tratamentos com rebento de março (3 RM) e o rebento de maio (4 RMa), ficaram um período somente com a planta matriz ,fato que deve ter proporcionado melhores condições de produção de folhas, pela menor competição ocorrida entre a planta matriz e o rebento.

O número de folhas desse ensaio foi maior do que o obtido por SAMPAIO (1967) e MATTOS (1969) por ocasião do aparecimento da inflorescência.

\section{Ciclo da produção}

A análise de variância para o número de dias decorridos do plantio à colheita do cacho (ciclo de produção), mostrou diferença significativa entre os tratamentos, ao nível de $1 \%$ de probabilidade.

Comparação das médias obtidas aparece no quadro 3.

Quadro 3 - Comparação das médias em relação ao ciclo de produção.

Média do número de dias

$5 \mathrm{~T}$

$4 \mathrm{RMa}$

$2 \mathrm{RJ}$

$1 \mathrm{SR}$

3 RM
468,4 a (*)

458,2 a b

450,8 a b

444,0 a b

$431,8 \quad b$

(*) As médias seguidas por letras diferentes apresentam diferença significativa ao nivel de $5 \%$ de probabilidade pelo teste de Tukey.

Não houve diferença significativa no que se refere ao ciclo de produção, entre os tratamentos $5 \mathrm{~T}, 4 \mathrm{RMa}, 2 \mathrm{RJ}$ e $1 \mathrm{SR}$; porém o tratamento $5 \mathrm{~T}$ foi diferente do tratamento $3 \mathrm{RM}$. Os tratamentos $4 \mathrm{RMa}, 2 \mathrm{RJ}, 1 \mathrm{SR}$ e $3 \mathrm{RM}$ não se diferenciaram entre si.

$\mathrm{O}$ tratamento testemunha, com todos os rebentos, (5 T) apresentou um ciclo mais longo, fato que pode ser explicado pela maior competição entre a planta matriz e seus rebentos. MISSINGHAN (1963) notou pouca diferença entre os diversos espaçamentos e ciclos de produção porém AHMED e MANNAN (1971), e MATTOS (1969), usando espaçamentos menores, obtiveram ciclo de produção maior. 


\section{Diâmetro do pseudo-caule na colheita do cacho}

A análise de variância não mostrou diferença significativa entre os 5 tratamentos, no que se refere ao diâmetro do pseudo-caule, na época da colheita do cacho. Estes resultados foram semelhantes aos obtidos por MATTOS (1969); entretanto, AHMED e MANNAN (1971), obtiveram diferenças significativas entre os espaçamentos menores e os espaçamentos maiores, com os espaçamentos menores apresentando menor diâmetro do pseudo-caule. No presente ensaio o menor diâmetro foi de $16,88 \mathrm{~cm}$, no tratamento $5 \mathrm{~T}$, e o maior foi de $18,70 \mathrm{~cm}$., nos tratamentos $3 \mathrm{RM}$ e $4 \mathrm{RMa}$, enquanto no trabalho de MATTOS (1969), os diâmetros variaram de 17,9 a $18,5 \mathrm{~cm}$.

\section{Número de folhas na época da colheita do cacho}

A análise de variância mostrou diferença significativa entre os tratamentos, ao nível de $1 \%$ de probabilidade, com relação ao número de folhas na colheita do cacho.

\section{Comparação das médias aparece no quadro 4}

Quadro 4 - Comparação das médias do número de folhas na colheita do cachos.

\section{Tratamentos}

Média do número de folhas

4 RMa

$1 \mathrm{SR}$

3 RM

$2 \mathrm{RJ}$

$5 \mathrm{~T}$

$$
\begin{array}{llll}
7,40 & \text { a } & (*) \\
6,42 & \text { a } & \text { b } \\
6,12 & \text { a } & \text { b } \\
4,28 & \text { b } & c \\
2,38 & & c
\end{array}
$$

(*) As médias seguidas por letras diferentes apresentam diferença significativa ao nivel de $5 \%$ de probabilidade pelo teste de Tukey

Pela comparação das médias, os tratamentos $4 \mathrm{RMa}, 1 \mathrm{SR}$ e $3 \mathrm{RM}$ foram semelhantes entre si, em relação ao número de folhas na colheita do cacho; porém, o tratamento $4 \mathrm{RMa}$ foi diferente dos tratamentos $2 \mathrm{RJ}$ e $5 \mathrm{~T}$. Os tratamentos $1 \mathrm{SR}$ e $3 \mathrm{RM}$ apresentaram diferença significativa em relação ao tratamento $5 \mathrm{~T}$. Os tratamentos $2 \mathrm{RJ}$ e $5 \mathrm{~T}$ foram semelhantes entre si.

Os tratamentos: planta com rebento de maio (4 $\mathrm{RMa}$ ), planta com rebento de março (3 RM) e planta sem rebento (1 SR), apresentaram maior número de folhas normais por ocasião da colheita do cacho, o que pode ser considerado um resultado esperado, porque, permanecendo durante todo $o$ tempo sem rebento ( $1 \mathrm{SR}$ ), e com o surgimento dos rebentos mais tarde 
(3 $\mathrm{RM}$ e $4 \mathrm{RMa}$ ) houve menor competição entre a planta matriz e o rebento, enquanto que no tratamento testemunha, com todos os rebentos (5 T) e maior número de rebentos nascidos mais cedo, deve ter ocorrido uma maior competição entre a planta matriz e seus rebentos.

\section{Peso do cacho}

A análise de variância para peso do cacho mostrou diferença significativa entre os tratamentos, ao nível de $5 \%$ de probabilidade.

0 quadro 5 mostra a comparação das médias de peso do cacho.

Quadro 5 - Comparação das médias do peso do cacho.

\begin{tabular}{lc}
\hline Tratamentos & Média do peso do cacho \\
\hline $1 \mathrm{SR}$ & $12,56 \mathrm{a}\left(^{*}\right)$ \\
$3 \mathrm{RM}$ & $12,10 \mathrm{a}$ \\
$4 \mathrm{RMa}$ & $11,80 \mathrm{a}$ \\
$2 \mathrm{RJ}$ & $9,58 \mathrm{a} \mathrm{b}$ \\
$5 \mathrm{~T}$ & $8,56 \mathrm{~b}$ \\
\hline
\end{tabular}

(*) As médias seguidas por letras diferentes apresentam diferença significativa ao nivel de $5 \%$ de probabilidade, pelo teste de Duncan.

Pela comparação das médias, os tratamentos 1 SR, $3 \mathrm{RM}, 4 \mathrm{RMa}$ e $2 \mathrm{RJ}$ não mostraram diferença significativa entre si em relação ao peso do cacho, porém os tratamentos $1 \mathrm{SR}, 3 \mathrm{RM}$ e $4 \mathrm{RMa}$ mostraram diferença significativa quando comparados com o tratamento $5 \mathrm{~T}$. Os tratamentos $2 \mathrm{RJ}$ e $5 \mathrm{~T}$ foram equivalentes entre si.

$\mathrm{O}$ tratamento $1 \mathrm{SR}$ permaneceu somente com a planta matriz enquanto nos tratamentos $3 \mathrm{RM}$ e $4 \mathrm{RMa}$, o nascimento do rebento ocorreu mais tarde e devendo ter ocorrido menor competição com a planta matriz. No tratamento testemunha ( $5 \mathrm{~T}$ ) que permaneceu com todos os rebentos que nasceram, deve ter ocorrido competição entre a planta matriz e os demais rebentos ocorrendo como consequência produção de cachos menores.

Os trabalhos de MATTOS (1969), AHMED e MANNAN (1971) e MISSINGHAN (1963) obtiveram menores espaçamentos produzindo cachos menores.

BHAN e MAZUNDER (1961), para a variedade Mantaman e Nanica, obtiveram cachos menores com espaçamentos menores, a não ser para a variedade Champa, na qual não houve influência do espaçamento sobre o tamanho do cacho, resultados que também foram obtidos por JAGIRDAR ef alii (1964). 
As médias dos pesos dos cachos obtidos no presente ensaio foram muito menores do que as obtidas por MATTOS (1969). Acontece que a época de saída da inflorescência e desenvolvimento dos frutos, neste ensaio, coincidiu com a época seca do ano (menor teor de água no solo) ocorrendo temperaturas muito baixas (mínima de $3^{\circ} \mathrm{C}$ em junho e $0,2^{\circ} \mathrm{C}$ em julho de 1972 ), fatores que são considerados negativos para obtenção de cachos de bananas com bom tamanho e peso.

\section{Número de pencas e frutos por cacho.}

A análise de variância não mostrou diferença significativa entre os tratamentos, com relação ao número de pencas e frutos por cacho.

Os resultados do presente ensaio concordam com os obtidos por MATTOS (1969), em relação ao número de pencas e com BHAN e MAZUNDER (1961), em relação ao número de pencas e frutos.

O número médio de pencas ,neste experimento, foi de 7,78; 7,62 e 7,30 para os tratamentos $1 \mathrm{SR} ; 3 \mathrm{RM}$ e $4 \mathrm{RMa}$ respectivamente; resultados muito semelhantes aos obtidos por SAMPAIO (1967): 7,3 e por MATTOS (1969): 7,5.

$\mathrm{O}$ número de frutos por cacho variou de 91 frutos para o tratamento $5 \mathrm{~T}$ a 115 frutos para o tratamento 1 SR.

\section{CONCLUSOES}

Os resultados obtidos neste trabalho permitem tirar as seguintes conclusões:

1. Com relação ao número de dias do plantio ao florescimento o tratamento planta matriz com rebento de maio ( $4 \mathrm{RMa}$ ) apresentou um ciclo maior do que os tratamentos planta matriz com rebento de janeiro (2 RJ), planta matriz sem rebento (1 SR) e planta matriz com rebento de março ( $3 \mathrm{RM}$ ). $O$ tratamento testemunha, com todos os rebentos (5T), apresentou um ciclo maior do que o tratamento planta matriz com rebento de março (3 RM).

2. Os tratamentos com rebento de março (3 RM), sem rebento (1 SR) e rebento de maio ( $4 \mathrm{RMa}$ ), apresentaram número de folhas maior na época do florescimento do que os tratamentos com rebentos de janeiro ( $2 \mathrm{RJ}$ ) e a testemunha com todos os rebentos (5 T).

3. O tratamento com rebento de março (3 $\mathrm{RM}$ ) apresentou um ciclo de produção menor do que o tratamento testemunha, com todos os rebentos (5 T). Os demais tratamentos foram semelhantes entre si.

4. O tratamento com rebento de maio (4 RMa), teve maior número de folhas na época de colheita do cacho do que os tratamentos com rebentos de janeiro $(2 \mathrm{RJ})$ e a testemunha com todos os rebentos $(5 \mathrm{~T})$. Os tratamentos sem rebento (1 SR) e com rebento de março (3 RM) apresentaram maior número de folhas que o tratamento testemunha, com todos os rebentos (5 T).

5. O peso do cacho dos tratamentos sem rebento, com rebento de março 
(3 $\mathrm{RM}$ ) e com rebento de maio (4 RMa) foi superior ao do tratamento testemunha com todos os rebentos $(5 \mathrm{~T})$.

6. Não houve diferença significativa entre os diversos tratamentos em relação ao diâmetro do pseudo-caule no florescimento e época de colheita do cacho, número de pencas e frutos por cacho.

\section{SUMMARY}

THE INFLUENCE OF SELECTION TIME OF SUCKERS ON THE DEVELOPMENT OF PLANT MATRIX IN BANANA (MUSA CAVENDISH L. NANICÃO).

Th objective of this study was to determine the effects of selection time suckers on the growth and production of banana matrix plant (Musa cavendishii Lamb) cv, Nanicão.

The trial was carried out at the Escola Superior de Agricultura «Luiz de Queiroz», Piracicaba (SP) Brazil, from september, 19, 1971, to January 31, 1973.

A randomized blocks design was used with five treatments and five replications

The treatments were as folows:

1. Matrix plant whitout suckers;

2. Matrix plant with January sucker;

3. Matrix plant with March sucker;

4. Matrix plant with may sucker; and

5. Control matrix plant with all suckers.

The results of study were:

a. Matrix plant with May sucker incresead the time from planting to flowering as compared with January sucker, no sucker, and March sucker; control matrix plant with all suckers delayed flowering time as compared with matrix plant witch March sucker.

b. The pseudostem circunference at flowering time were similar in all treatments except treatment with January sucker and control matrix plant with all suckers; these treatments have resulted in a markerd reduction in circunference of pseudostem at flowering time.

c. Production cycle periods were similar for treatments no sucker, January and May sucker; the production cycle was smaller with March sucker and larger in control matrix plant with all suckers.

d. The number of leaves in harvesting time was highest in May sucker as compared with January sucker and control matrix plant with all suckers; the treatments no sucker and March sucker had highest number of leaves as compared control matrix plant with all suckers. 
e. Control matrix plant with all suckers had lower average bunch weight as compared with treatments with no sucker, March and May sucker.

f. These was no significance diference on pseudostem circunference at flowering and harvesting time, «hands» and «finger» number per bunch.

\section{LITERATURA CITADA}

AHMED, K. \& MANNAN, A. Effect of size of pit and spacing on the Amaritsagor banana. Punjab fruit. J. 32(110/111): 7.13. 1970. In: Hort. Abstr. 41(2): 599. Abstr. 4992. 1971.

AUBERT, B. Action du climat sur les comportement du bananier em zones tropicales et subtropicales. Fruits, Paris, 26(3): 175-188. 1971.

BERRIL, F. W. Spacing bananas. Od. Agric. J., Queensland 89: 35-8. 1963.

BHAN, K, C. \& MAZUNDER, P. K., Spacing trials on banana in West Bengal. Indian J. Agric. Sci., $31: 1491-55.1961$.

BORGES O. L., Estúdio comparativo de diferentes clones de platano y cambures. Agron. Trop., Maracay 21(4) : 265-275. 1971.

CHAMPION, J. Le Bananier. Paris, Maisonneuve et Larose, 1963. $264 \mathrm{p}$.

CORTEZ, J. V. Contribuição para o estudo da bananeira no litoral do Estado de São Paulo. Observaçôes sobre ciclos. Piracicaba, ESALQ, 1961, 46 p. (Tese para Doutoramento).

F. A. O. Production Yearbook. Roma, FAO, 1970. 822 p.

I.B.G.E. Anuário Estatístico do Brasil. Rio de Janeiro, Fundação IBGE, 1972. $989 \mathrm{p}$.

JAGIRDAR, P.A.P., BHUTO, M. A. \& SHAIK, A. M. Effect of spacing, interval of irrigation and fertilizer application of Basrai banana (Musa cavendishii Lamb) W. Pakist. J. Agric. Res. 1(2) : 5-20. 1963. In Hort. Abstract. 34(2): 381 Abstr. 3691. 1964.

KERVEGANT D. Le bananier et son explotation. Paris, Societé d'Editions Geographiques, Maritimes et Coloniales, 1935. 578 p.

MATTOS, J. R. Aspectos da densidade do bananal no litoral do Estado de São Paulo. Piracicaba, ESALQ. 1969. 71 p. (Tese de Doutoramento).

MISSINGHAN, L. J. Closer spacing gives more bananas in North Queensland. Od., Agric. J., Queensland, 89: 676-9. 1963.

RANZANI, G. FREIRE, O. \& KINJO, T. Carta de solos do Municipio de Piracicaba. Piracicaba, ESALQ, 1966. 85 p.

RODRIGUES A. \& SOUZA, A. T. de. Sobre a época de seleção dos rebentos da bananeira (Musa nana Lour.) seu desenvolvimento e frutificação na Ilha de Madeira. Agron. Lusitana, Oeiras, 9(2): 193-248. 1947.

SAMPAIO, V. R. Banana. Estudo comparativo das variedades "Nanicão" $e$ "nanica" no Litoral do Estado de São Paulo. Piracicaba, ESALQ, 1967. 71 p. (Tese de Doutoramento).

WARDLAW, C. W., Banana diseases including plantains and abaca. London, Longmans, 1961. $648 \mathrm{p}$. 